\title{
Una Vida entre Rejas: Aspectos Psicosociales de la Encarcelación y Diferencias de Género
}

\section{A Life behind Bars: Psychosocial Aspect of Incarceration and Gender Differences}

\author{
M. Carmen Herrera Enríquez y Francisca Expósito Jiménez \\ Universidad de Granada - España
}

\begin{abstract}
Resumen. Dado el rol que la mujer tiene en la familia, su ingreso en prisión conlleva un estigma familiar, alteración de roles y problemas de apego, efectos que se ven agravados en el caso de tener hijos pequeños en prisión. El propósito de esta investigación es contrastar las diferencias, entre las respuestas que dan hombres y mujeres a una serie de cuestiones referidas a su paso por la cárcel. Concretamente, conocer si el paso por la institución afecta de manera diferencial a unos y otras y analizar las diferencias a la luz de las teorías sobre roles de género. Es por ello que resulta de suma importancia atender el hecho de que no se trata de que las mujeres tengan problemas diferentes a los que pueden hallarse en los hombres, sino que a las consecuencias propias que se derivan del encierro en hombres y mujeres, se suman las propias de la condición de género.

Palabras clave: delincuencia femenina, género, roles, encarcelación.
\end{abstract}

\begin{abstract}
Given the role that women play in the family, their imprisonment brings about a stigma in the family, an alteration of roles and attachment problems. These effects worsen for those who have young children while in prison. The purpose of this research is to contrast the differences between men's and women's responses to certain issues related to the time they spent in prison. Specifically, the purpose is to analyze whether time in prison affects the genders in different ways and to analyze any differences in the light of gender role theories. It is of utmost importance to address the fact that it is not that women have different problems than those which can be found in men, but rather that the consequences arising from incarceration for men and for women come on top of those differences already inherent to the genders.

Keywords: female crime, gender, roles, incarceration.
\end{abstract}

La delincuencia femenina ha sido una cuestión generalmente ignorada hasta hace muy poco tiempo, y en parte, esta ignorancia se ha debido a la creencia de que los delitos cometidos por las mujeres sólo afectaban al ámbito doméstico, y la sociedad, por tanto, no se veía amenazada por este tipo de conductas (Cecil, 2007; Lorenzo, 2002). Desde que los roles de la mujer empiezan a aumentar, su comportamiento también tiene trascendencia en la vida pública, pero poco se ha divulgado, hasta la fecha, de las características específicas actuales de la delincuencia femenina. La mayoría de estudios, investigaciones y debates, siguen centrándose en la población mayoritariamente masculina, ya que la criminalidad se asocia culturalmente con la masculinidad (Lombroso y Ferraro, 1895; Pollack, 1950). Se suele alegar para justificarlo, la insignificancia de las cifras de delincuencia femenina respecto a la masculina, en España, el $91.98 \%$ de los presos son hombres, frente al $8.02 \%$ que son mujeres (Institucio-nes penitenciarias, 2010). Las principales teorías que se

La correspondencia sobre este artículo puede dirigirse a Mari Carmen Herrera Enríquez. Departamento de Psicología Social y Metodología de las Ciencias del Comportamiento. Facultad de Psicología, Universidad de Granada. Campus Cartuja s/n. 18071 Granada (Spain). han esbozado para explicar la menor criminalización de las mujeres pueden resumirse del siguiente modo (Romero y Aguilera, 2002):

Las mujeres delinquen menos que los hombres: Esta teoría entiende que las mujeres se comportan conforme a la ley en mayor medida que los hombres. Esta afirmación ha sido explicada, en primer lugar, a través del rol cultural atribuido a las mujeres en la sociedad (pasividad, obediencia, cuidado), y en segundo lugar, a través de causas de tipo biológico. Esta teoría se ha desarrollado y hoy día es difícil mantener que son causas de tipo biológico las que explican las diferencias de género en delincuencia. Más bien, parece existir una cierta relación entre la tasa de criminalidad femenina y la incorporación de las mujeres al mercado laboral, de manera que a medida que la mujer se ha ido incorporando de lleno en el mundo laboral y sus oportunidades han sido similares a las del hombre, estas diferencias en delincuencia, también han cambiado. Sin embargo, esta relación se pone en entredicho al observar que los

Agradecimientos: Esta investigación ha sido posible gracias al permiso concedido por Instituciones Penitenciarias y se enmarca dentro del proyecto Exp. No. 064/07 concedido por el Instituto de la Mujer. 
países europeos donde las mujeres han logrado una mayor emancipación económica (Holanda, Austria, Noruega, Finlandia) son los que presentan un índice menor de criminalidad femenina.

Las mujeres delinquen en la misma medida que los hombres, pero obtienen trato "de favor" en el proceso judicial. Sin embargo, la "caballerosidad" del sistema penal ha sido rebatida con diversos estudios sobre la discriminación por razón de sexo en el sistema judicial. En este sentido resulta concluyente un estudio de Fernández Villanueva, Fernández Cantero y Orto Poveda (1988), según el cual una de las razones de la mayor dureza de la respuesta judicial ante las mujeres puede ser que el delito supone, en el caso de éstas, una doble contravención, por una parte de la ley escrita, pero por otra (la más imperdonable) la del rol social de sumisión y obediencia que le ha sido asignado.

En general, la cárcel esta construida con una concepción androcéntrica, es decir el sistema penitenciario (sus normas, prácticas, roles y representaciones) ha sido elaborado por hombres y para hombres. Se podría afirmar que los conflictos de las mujeres privadas de libertad son similares a los de cualquier mujer que se encuentra en libertad, porque dentro y fuera de las cárceles está condenada a cumplir con los roles asignados a su género.

Hasta este momento, la exclusión social es el factor explicativo determinante de la inmersión de la mujer en el mundo delictivo actual, pues numéricamente, desde los años 50 hasta mediados de la década de los 80 , la incidencia de la mujer en prisión era muy escasa, coincidiendo con el poco peso de su papel social y debido al fuerte control ejercido por las estructuras sociales y familiares (Yaguie y Cabello, 2005).

\section{Núcleo familiar}

Cuando una mujer entra en prisión no se producen las mismas consecuencias que cuando lo hace un hombre. Las mujeres sufren más la prisión que los hombres, ya que su encarcelación afecta directamente al núcleo familiar, ellas suelen ser el soporte de la familia, tanto afectivo como económico. Las mujeres encarceladas sufren una doble condena: la del encierro y la social. Las mujeres tienen un papel central en el mantenimiento de la familia, ello les lleva a trabajar, tanto fuera como dentro de casa, combinando y haciendo compatibles las tareas del hogar, la crianza de los hijos e hijas y el trabajo fuera de casa. En el momento de ser encarceladas suelen desempeñar un papel de gran responsabilidad en el ámbito familiar, por lo que el cumplimiento de largas condenas por parte de estas mujeres está teniendo además, consecuencias para muchas más personas.

El ingreso en prisión de una mujer conlleva el estigma familiar, alteración de los roles y problemas de apego que se ven agravados en el caso de tener hijos e hijas pequeños en prisión (Lagarde, 1993). Para estas madres, resulta muy necesaria una red social que las provea de apoyo emocional, para así prevenir situaciones estresantes, inevitables en gran medida, a las que han de enfrentarse (López Verdugo et al., 2007).

\section{Prisionización}

Aunque la privación de libertad parece la consecuencia más obvia del encarcelamiento, existen otras muchas dimensiones que se ven afectadas tanto de la propia persona presa como de otras personas. A partir de investigaciones mayoritariamente realizadas en prisiones norteamericanas, la prisionización ha sido concebida en términos de la asimilación por los internos de hábitos, usos, costumbres, y cultura de la prisión, así como una disminución general del repertorio de conducta de los mismos, por efecto de su estancia prolongada en el centro penitenciario (Clemmer, 1940; Goffman, 1979; Pérez y Redondo, 1991; Pinatel, 1969). Estos efectos tendrían lugar tanto durante el período del encarcelamiento de los sujetos como en su posterior vida en libertad. Entre los efectos más destacables de la prisionización se encontrarían los siguientes:

1. Un aumento del grado de dependencia de las personas encarceladas, debido al amplio control conductual a que se ven sometidas. La mayoría de las decisiones que afectan a su vida diaria les son impuestas, escapando a su propio control. Si evaluamos el locus de control, atribuirían la causa de su propio comportamiento a factores externos (Pérez y Redondo, 1991; Rotter, 1966).

2. Devaluación de la propia imagen y disminución de la autoestima, concebidas como la valoración que el individuo realiza y mantiene respecto de si mismo (Coopersmith, 1959). En términos generales, se ha sugerido que el «sistema social informal» de la prisión tiene efectos negativos en la autoestima y la autopercepción de los sujetos (Smith y Hogan, 1973). En el caso de la autoestima, se sigue un proceso de "U" normal, de manera que el simple hecho de ingresar en la cárcel reduce la autoestima, pero conforme va pasando el tiempo, la persona va reconstruyendo el autoconcepto a través de la valoración que hacemos de nosotros mismos. Con el paso del tiempo los niveles de autoestima son similares a los que tenia cuando ingresó, pero es una autoestima totalmente diferente, los referentes son otros. El proceso de prisionización sigue un proceso de "U" invertida, es decir, a medida que va pasando el tiempo la persona tiene que aprender a aceptar su nueva vida, cuando la prisionización es máxima es cuando la autoestima mas baja está (Pérez y Redondo, 1991).

Desde una perspectiva de género, el fenómeno de la prisionización femenina impone actividades destinadas a reafirmar el rol genérico asignado social y culturalmente a la mujer. 
Una de las afirmaciones más repetidas entre las personas encarceladas es que lo que peor llevan del encierro es la separación forzada de sus familias. Al producirse, generalmente de manera inesperada la detención y posterior ingreso en prisión, la persona se siente arrancada del entorno familiar y de los suyos.

La gama de repercusiones sobre la estabilidad familiar es muy variada, dependiendo de la estructura previa, el tiempo de condena y las expectativas de unos y otros acerca de la relación. Estas pueden ser irreparables cuando se produce la adopción de los hijos por terceros, o el abandono y la creación de una nueva familia por parte del cónyuge que queda en libertad.

Resulta de suma importancia resaltar que no se trata de que las mujeres tengan problemas diferentes a los que pueden tener los hombres encarcelados (sobrepoblación, hacinamiento, falta de recursos, etc.), sino que al conjunto de afectaciones comunes que puedan tener hombres y mujeres en el encierro, se suman las propias de la condición de género. Estas mujeres sufren una doble discriminación, son discriminadas por ser mujeres y por ser reclusas.

Con esta investigación se pretenden los siguientes objetivos:

1. Contrastar las diferencias, si las hubiera, entre las respuestas que dan hombres y mujeres a una serie de cuestiones referidas a su paso por la cárcel.

2. Conocer si el efecto de la institución afecta de manera diferencial a unos y otras, y analizar las diferencias de género.

3. Centrándonos en la muestra de mujeres, analizar qué áreas de su vida se ven más afectadas por su paso por prisión (ámbito familiar, social, etc.).

La principal hipótesis de este estudio se apoya en que las mujeres se verán más afectadas debido al rol que ocupan en la sociedad y en la familia.

\section{Método}

Este estudio ha sido realizado con un total de 291 reclusos internados en distintas cárceles andaluzas. El $58.1 \%$ fueron hombres y el $41.9 \%$ mujeres. La media de edad fue 35.55 ( $S D=8.49)$ oscilando entre los 19 y 72 años.

\section{Procedimiento}

Todos los participantes accedieron a contestar de manera anónima e individualizada un cuestionario, en presencia de alguno de los psicólogos que participaron en el estudio. Estos aclararon personalmente cualquier duda o problema de comprensión que hubiera surgido durante la aplicación del cuestionario. El estudio se desarrolló tras autorización previa y con la colaboración plena de las administraciones penitenciarias implicadas.

\section{Instrumentos}

1. Características sociodemográficas: edad, duración de la condena, lugar de procedencia, raza, delito cometido, tiempo que lleva en prisión, tiempo que le queda para salir, nivel de estudios, profesión, religiosidad, situación de pareja, número de hijos, etc.

2. Índice de satisfacción con las visitas y actividades (elaboración propia): consta de 3 ítems que medían su nivel de satisfacción en las visitas recibidas en locutorios, vis a vis y con las actividades realizadas. El formato de respuesta es una escala tipo Likert de 4 puntos (1-nada satisfecho4-muy satisfecho)

3. Índice de preocupación y efectos del encarcelamiento (elaboración propia). Se les preguntaba por las áreas que consideraba se verían más afectadas (familia, pareja, hijos), y lo puntuaban a en una escala tipo Likert de 4 puntos (1-nada afectada-4-muy afectada).

4. Escala de Autoestima de Rosenberg (1965). Consta de 10 ítems (5 formulados de forma positiva y 5 negativa) y el formato de respuesta es tipo Likert de 4 puntos (desde muy de acuerdo hasta muy en desacuerdo). Conforme mayor es la puntuación, menor es la autoestima. El coeficiente alpha para nuestra muestra fue de .76 .

\section{Resultados}

\section{Características de la población estudiada}

En primer lugar se realizaron una serie de análisis descriptivos para obtener información sobre las principales características sociodemográficas de la población objeto de estudio. Los resultados pueden observarse en la tabla 1.

Tabla 1.Lugar de procedencia y raza de la población objeto de estudio, diferenciadas por género

\begin{tabular}{lcc}
\hline Lugar procedencia & Hombres & Mujeres \\
\hline España & $81.8 \%$ & $73.7 \%$ \\
Sudamérica & $4.2 \%$ & $8.5 \%$ \\
Europa Este & $1.8 \%$ & $4.2 \%$ \\
Asia & $0.6 \%$ & 0 \\
África & $8.5 \%$ & $4.2 \%$ \\
Otro & $3 \%$ & $9.3 \%$ \\
\hline Raza & & \\
\hline Blanca & & \\
Gitana & $80.5 \%$ & $58.1 \%$ \\
Negra & $15.9 \%$ & $29.1 \%$ \\
Otra & $0.6 \%$ & $5.1 \%$ \\
\hline
\end{tabular}


También resultaba de máximo interés conocer el tipo de delito por el que estaban cumpliendo condena, así como las posibles diferencias existentes entre hombres y mujeres, estos datos se pueden ver en la tabla 2 .

Tabla 2. Tipo de delito por el que cumplen condena, hombres y mujeres

\begin{tabular}{lrr}
\hline Delito & Hombres & Mujeres \\
\hline España & $81.8 \%$ & $73.7 \%$ \\
Salud Pública & $30 \%$ & $63 \%$ \\
Robo contra propiedad & $24.2 \%$ & $12.7 \%$ \\
Violencia de Género & $12.7 \%$ & $0 \%$ \\
Robo con violencia & $21.2 \%$ & $9.3 \%$ \\
Delito de lesiones & $9.7 \%$ & $3.4 \%$ \\
Homicidio & $4.8 \%$ & $1.7 \%$ \\
Otro & $12.8 \%$ & $16.9 \%$ \\
\hline
\end{tabular}

\section{Percepción de satisfacción}

Tras realizar un análisis de varianza, usando como variables dependientes la satisfacción con las visitas y actividades y como factor el sexo del participante, se encontraron diferencias marginalmente significativas en cuanto al nivel de satisfacción de unos y otras con las visitas recibidas en los locutorios $F(1,220)=2.75, p=.09$. Sin embargo, no se encontraron diferencias significativas en el nivel de satisfacción con las visitas vis a vis $F$ (1, $234)=.399, n . s$; ni con la satisfacción por la realización de actividades $F(1,263)=.141, n . s$; ni con la satisfacción total $F(1,251)=.267$, n.s. Aunque la tendencia indica que las mujeres suelen estar más satisfechas que los hombres, como puede observarse en la figura 1.

\section{Efecto de la prisionización}

Para comprobar si existían diferencias entre hombres y mujeres en su percepción del efecto que el paso por prisión tendrá en distintas áreas de su vida, realizamos un análisis de varianza, usando como variables dependientes las posibles áreas que se veían afectadas, y como factor, el sexo del participante. Encontramos diferencias significativas en las siguientes áreas: pareja $F(1,169)=$ $9.68, p<.01$; relación con los amigos $F(1,240)=16.07$, $p<.001$; y las relaciones sociales en general $F(1,267)=$ $5.79, p<.01$. No se encontraron diferencias significativas en la relación con la familia $F(1,263)=0.86$, n.s. De manera que las mujeres creen que la relación con sus amigos y sus relaciones sociales en general se verán afectadas, en mayor medida que los hombres. Mientras que éstos creen, más que las mujeres, que su relación de pareja se verá afectada por su ingreso en prisión, como puede observarse en la figura 2.

\section{El papel de la maternidad}

Por último, nos resultó interesante centrarnos sólo en la población reclusa femenina y observar si existen diferencias entre las mujeres que tienen hijos y las

Figura 1. Nivel de satisfacción de hombres y mujeres con las visitas y actividades

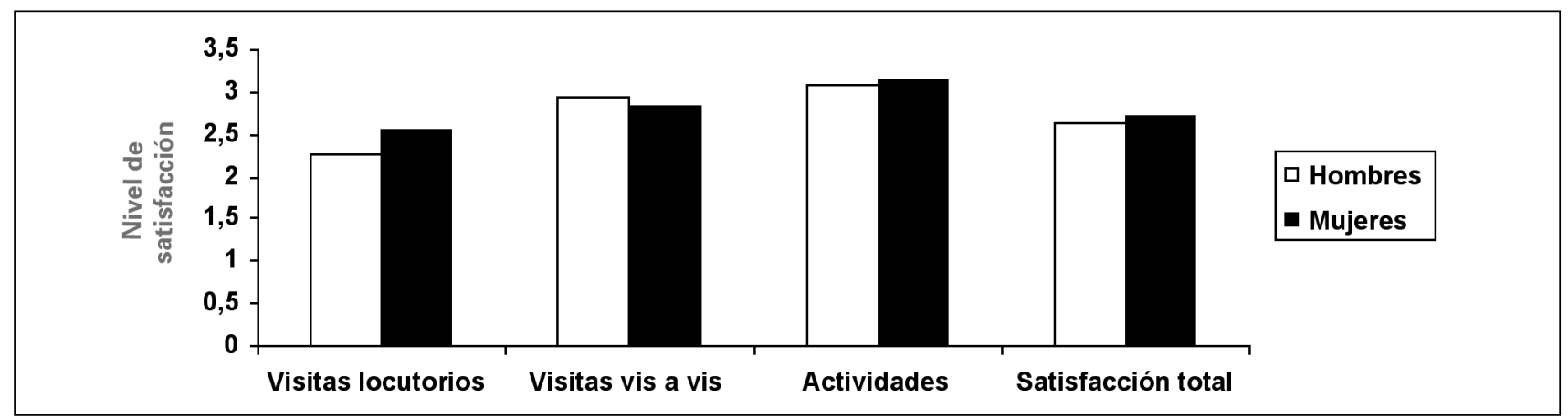

Figura 2. Percepción del efecto que el paso por prisión tendrá en distintas áreas de su vida

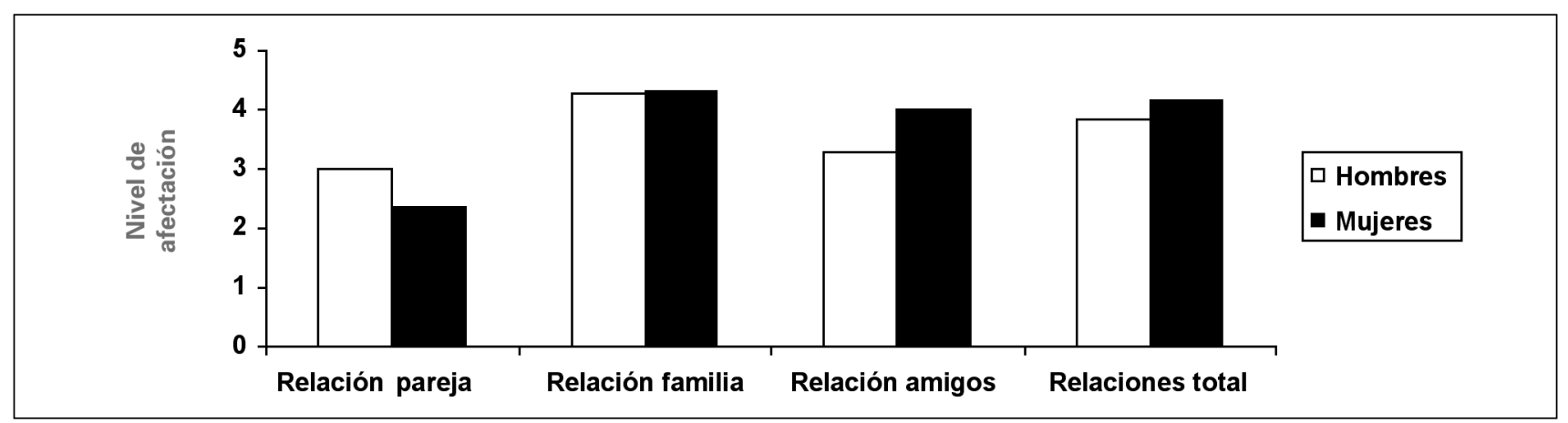


que no tienen, para comprobar si el rol de madre se resiente por su paso por la cárcel. Se realizó un análisis de varianza utilizando como variables dependientes las puntuaciones obtenidas en las distintas medidas (principales áreas afectadas y autoestima), con el factor tipo de mujer (con hijos o sin hijos). Como se puede observar en la figura 3 , se encontraron diferencias significativas entre las mujeres con hijos y las que no tienen hijos en el grado en que piensan que la familia se ve afectada por su paso por prisión $F(1$, $89)=5.97, p<.01$; así como en los niveles de autoestima $F(1,105)=4.73, p<.05$. De manera que las mujeres sin hijos, creen en mayor medida que la familia se verá muy afectada por su paso por prisión, presentando también una menor autoestima que las mujeres con hijos. asociados sólo con hombres, por la violencia implícita que conlleva su ejecución. No obstante, según reflejan nuestros datos, los delitos que cometen las mujeres están relacionados con la salud pública y otros más encubiertos entre los que podrían encontrarse la prostitución. En ambos casos delitos relacionados con la necesidad de supervivencia.

Pese a que no contamos con el dato empírico, como resultado de las conversaciones mantenidas durante la recogida de datos en los diferentes centros, podemos afirmar que las diferencias de género existen y que en general, los hombres tienden a justificar más los delitos que comenten, se perciben como víctimas del sistema, suelen hacer atribuciones externas y por tanto se creen con derecho a exigir mejores condiciones penitenciarias, y a no asumir su responsabilidad del hecho

Figura 3. Principales áreas afectadas en mujeres con hijos vs. mujeres sin hijos

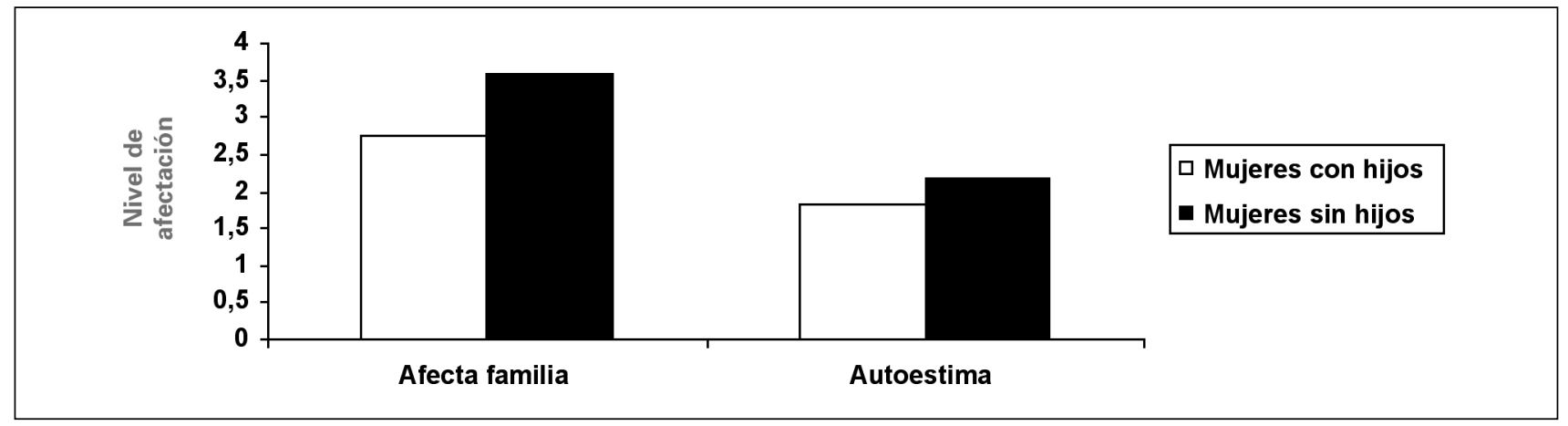

\section{Discusión}

La realidad de nuestras cárceles ha cambiado en los últimos años. Si hace unas décadas lo frecuente era encontrar en ellas personas desadaptadas socialmente y que reunían las características típicas de la exclusión social, hoy día debido a los cambios sociales y legislativos, así como al endurecimiento de las penas encontramos una población mucho más heterogénea.

En el caso concreto de la población reclusa femenina, cabe señalar el incremento en las cifras de este colectivo, así como que está compuesto principalmente por jóvenes marginadas, inmigrantes y gitanas, mujeres pertenecientes a grupos minoritarios y que han vivido en la pobreza la mayor parte de sus vidas. A diferencia de los hombres, la mujer que delinque, salvo en contadas ocasiones, no suele representar un gran riesgo social. La mayoría de las mujeres encarceladas provienen de sectores de la población económica y socialmente desfavorecidos y permanecen encarceladas por delitos típicos de personas que carecen de poder, han vivido en la pobreza y han sido violentadas la mayor parte de sus vidas (Chesney-Lind y Pasko, 2004). Sin embargo, recientemente, las mujeres se han visto involucradas en nuevas actividades delictivas como son asalto a banco, secuestro, extorsión y delitos contra la salud. Delitos que hasta hace poco, estaban delictivo. Las mujeres, por el contrario muestran el patrón opuesto, tienden a recriminarse su comportamiento, se sienten culpables de lo que han hecho, y probablemente por eso se muestran menos exigentes, sobrevaloran lo que tienen porque asumen la responsabilidad de sus actos, situación que les lleva a valorar el ambiente que les rodea de una manera menos hostil que la que esperábamos encontrar.

No existe duda de la importancia que tiene el apoyo social recibido o percibido ante los eventos negativos de la vida. En este caso, la percepción que tienen las mujeres de sus redes de apoyo es más positiva que la que tienen los hombres. Ésta puede ser una de las razones por las que las mujeres hacen una mejor valoración de su paso por la cárcel, ya que el apoyo social percibido podría estar actuando como amortiguador del hecho estresante que supone el paso por la cárcel.

Otro aspecto relevante, es la autoestima y el efecto que la prisionización tiene sobre ella. La autoestima de las mujeres proviene de los roles que desempeña, se trata pues de una autoestima derivada, por lo que cualquier cambio en los roles tradicionales (esposa y madre) afecta a la autoestima negativamente. La autoestima de los hombres se ve menos dañada que la de las mujeres, probablemente porque éstas últimas han desarrollado roles más familiares, más íntimos y su pérdida tiene un efecto negativo. Por otra parte, los 
hombres sienten menos el rechazo social que supone la delincuencia que las mujeres, a las que se les percibe de manera más negativa doblemente: por dejación de sus roles tradicionales, y por incorporar roles (negativos) tradicionalmente desempeñados por hombres.

En cuanto al efecto de los hijos, encontramos un resultado interesante: las mujeres que no tienen hijos tienen menor autoestima que las que si los tienen, contrario a lo esperado. Las mujeres sin hijos, cuando se les pregunta por la familia, suelen tener como grupo de referencia a padres y hermanos. Las que tienen hijos se centran en su propia familia, y posiblemente el tener hijos es un estímulo para sobrevivir en un ambiente hostil y un motivo suficientemente fuerte para seguir adelante. Pese a no tener datos empíricos, uno de los argumentos que mas utilizaban las mujeres para justificar su delito era el tener hijos a los que sacar adelante. Posiblemente, este hecho, el tener hijos, se convierte en la razón fundamental de sus vidas y ayude a relativizar la situación vital por la que están pasando.

En general, y pese a ser un ambiente no acorde a sus necesidades, las mujeres parecen mostrar una mayor capacidad de adaptación al contexto carcelario. Sus principales fuentes de apoyo son la familia y la pareja y el paso por la cárcel no deteriora sus relaciones tanto como esperábamos encontrar a priori y suelen percibir a su red de apoyo como más segura de lo que la perciben los hombres.

\section{Alternativas al encarcelamiento}

Con la reforma del código penal en 1996 se crearon algunas medidas nuevas cuyo propósito es evitar que la persona entre en prisión (sustitutivas) o que se pase el menor tiempo en ella (combinatorias). Hay que trabajar en esta dirección y poner a prueba todo tipo de mecanismos legales alternativos a la prisión: mujeres clasificadas en tercer grado que viven con sus hijos en casas en medio de la ciudad, otras que van sólo a dormir, el uso de las pulseras telemáticas, así como establecer programas alternativos que eviten el ingreso en prisión cuando sea posible. Se trata pues de habilitar los recursos necesarios para establecer "Unidades Dependientes"; Centros de Inserción Social (CIS) y Secciones Abiertas donde las mujeres puedan cumplir las penas en régimen abierto o semiabierto. Dado el coste anual que supone un recluso, aproximadamente unos 30.000 euros, y otros 20.000 por el cuidado de cada hijo, las organizaciones defensoras de las presas sostienen que las mujeres condenadas por delitos no violentos y que no supongan una amenaza a la sociedad, podrían recibir mejor tratamiento y menos costoso en centros de rehabilitación.

Por otra parte, la experiencia maternal y el sentimiento de responsabilidad hacia la familia es, generalmente, un efecto positivo de reinserción que garantiza la no reincidencia de este tipo de delincuentes, que, por lo general, se ven obligadas a mantener con ellas a sus hijos menores de 3 años. El hecho de que la mujer sea, generalmente, la principal cuidadora de la familia, sumado a que muchas mujeres que cometen delitos son las sustentadoras únicas de sus hijos, debería ser tenido en consideración a la hora de diseñar penas alternativas al encarcelamiento, más acordes a la realidad de este colectivo, y al objetivo último de la reinserción social.

\section{Referencias}

Cecil, D.K. (2007). Looking Beyond Caged Heat. Media Images of Women in Prison. Feminist Criminology, 2, 304-326.

Chesney-Lind, M. y Pasko, L. (2004). The female offender: Girls, women, and crime (2nd ed.). Thousand Oaks, CA: Sage.

Clemmer, P. (1949). The prison community. Boston: Cristopher Publishing Co.

Coopersmith, S. A. (1959). A Method for Determing Tipes of Self Esteem. Journal of Abnormal and Social Psychology, 59, 87-94.

Fernández Villanueva, C., Fernández Cantero, A., y Orto Poveda, P. (1988). La mujer ante la administración de Justicia. El caso del parricidio. Instituto de la Mujer: Madrid.

Goffman, E. (1979). Internados. Buenos Aires: Amotrortu Editores, 94.

Instituciones Penitenciarias (2010). Distribución de la población reclusa por sexo. Tomado de $h t t p: / / w w w$. institucionpenitenciaria.es, el 24 de Octubre de 2010.

Lagarde, M. (1993). Los cautiverios de las mujeres: madresposas, monjas, putas, presas y locas. México: UNAM.

Lombroso, C., y Ferraro, W. (1895). The female offender. London: Fisher Unwin.

López Verdugo, I., Menéndez Álvarez-Dardet, B., Lorente Lara, B., Jiménez García, L., Hidalgo García, M. V., y Sánchez Hidalgo, J. (2007). Evaluación del apoyo social mediante la escala ASSIS: descripción y resultados en una muestra de madres en situación de riesgo psicosocial. Intervención Psicosocial, 16, 323-337.

Lorenzo, M.M. (2002). La delincuencia femenina. Psicothema, 14, 174-180.

Pérez, E. y Redondo, S. (1991). Efectos psicológicos de la estancia en prisión. Papeles del Psicólogo, 48, 54-57.

Pinatel, J. (1969). La sociedad criminógena. Madrid: Aguilar.

Pollack, O. (1959). The Criminality of women. New Cork: AS Barnes / Perpetua.

Romero, M. y Aguilera, R. M. (2002). ¿Por qué delinquen las mujeres? Perspectivas teóricas tradicionales. Parte 1. Salud Mental, 25, 10-22. 
Rosenberg, M. (1965). Society and the adolescent selfimage. Princeton, NJ: Princeton University Press.

Rotter, J. B. (1966). Generalizad expectancies for internal versus external control of reinforcements. Psychological Monographs, 80 (whole No. 609).
Smith, J. C. y Hogan, G. (1973). Criminal Law. London: Burke worth, 94.

Yagüe, C. y Cabello, M.I. (2005). Mujeres jóvenes en prisión. Revista de estudios de juventud, 69, $30-48$.

Manuscrito Recibido: 22/06/2010

Revisión Recibida: 27/09/2010

Manuscrito Aceptado: 05/10/2010 